\title{
Swelling and Deswelling Kinetics of AT-O3S Polymer
}

\author{
Mahmoud Elsharafi and Cody Chancellor \\ McCoy School of Engineering College of Science and Mathematics, Midwestern State University, Wichita Falls, Texas 76310, United \\ States
}

Received: December 05, 2016 / Accepted: December 15, 2016 / Published: March 31, 2017.

\begin{abstract}
In the oil industry, techniques decreasing unwanted water production have drawn large amounts of interest from many companies. During water injection operations, water is injected into the oil reservoir to extract oil trapped in the formation. Due to the heterogeneity in the reservoir formation, oil production will decline and water production will increase as the injected water sweeps the high permeability zones. In order to flush out the oil remaining in the low permeability zones, many treatments have been used. One such treatment involves the injection of an SAP (superabsorbent polymer) into the high permeability zones. The swelled polymer will decrease the heterogeneity of reservoir permeability, thus forcing water injection into the oil rich, unswept zones/areas of the formation. Proper application of an SAP can have a dramatic impact on both the production and lifespan of mature oil wells. Successful treatment is reliant upon the reservoir salinity, temperature, and $\mathrm{pH}$.
\end{abstract}

Key words: Water shutoff, reservoir heterogeneity, swelling and deswelling of polymer, pH value, chemical treatment, enhanced oil recovery.

\section{Introduction}

As oil reservoirs mature, methods to increase oil extraction efficiency become increasingly important [1]. Water injection is a popular method used to artificially pressurize a permeable oil reservoir, allowing for continued production. Although it is a very useful technique, there are some drawbacks. Heterogeneity in reservoir permeability causes an increase in the amount of water produced over time [2]. This translates to greater production costs [3].

As the percentage of water increases, the percentage of produced oil must decrease. In extreme cases, wells may be producing $98 \%$ water [4]. The formation water has a high concentration of metal ions from salts present in a formation or resulting from injection water, with the two most common reservoir cations being Sodium $^{+}$ions and Calcium ${ }^{+2}$ ions, followed by Magnesium $^{+2}$ ions [5]. As a result, scaling and corrosion of equipment becomes a major issue, as does

Corresponding author: Mahmoud Elsharafi, Ph.D., assistant professor, research field: enhanced oil recovery. the processing and disposal of the brine mix [3]. The estimated cost of excess water production worldwide was reported around $\$ 40$ billion (USD) a year [6]. However, that was well over a decade before the writing of this document. Due to the large growth experienced in domestic (U.S.) drilling operations in recent years, the above estimation is most likely much less than the current costs incurred by excess water production.

Excess water production is often caused by differences in the permeability of a reservoir, which can affect fluid movement significantly $[2,7,8]$. Since injected water flows through the high permeability zones first, they are cleared of oil first, and water production begins to increase dramatically [9]. Although the reservoir may be producing small amounts of oil, a large reserve could remain in the unswept, low permeability zone [10]. Fig. 1 shows the deviation of water injection into the high permeability zone in a heterogeneous reservoir.

The challenge is to decrease reservoir heterogeneity, namely by decreasing the permeability of the high 


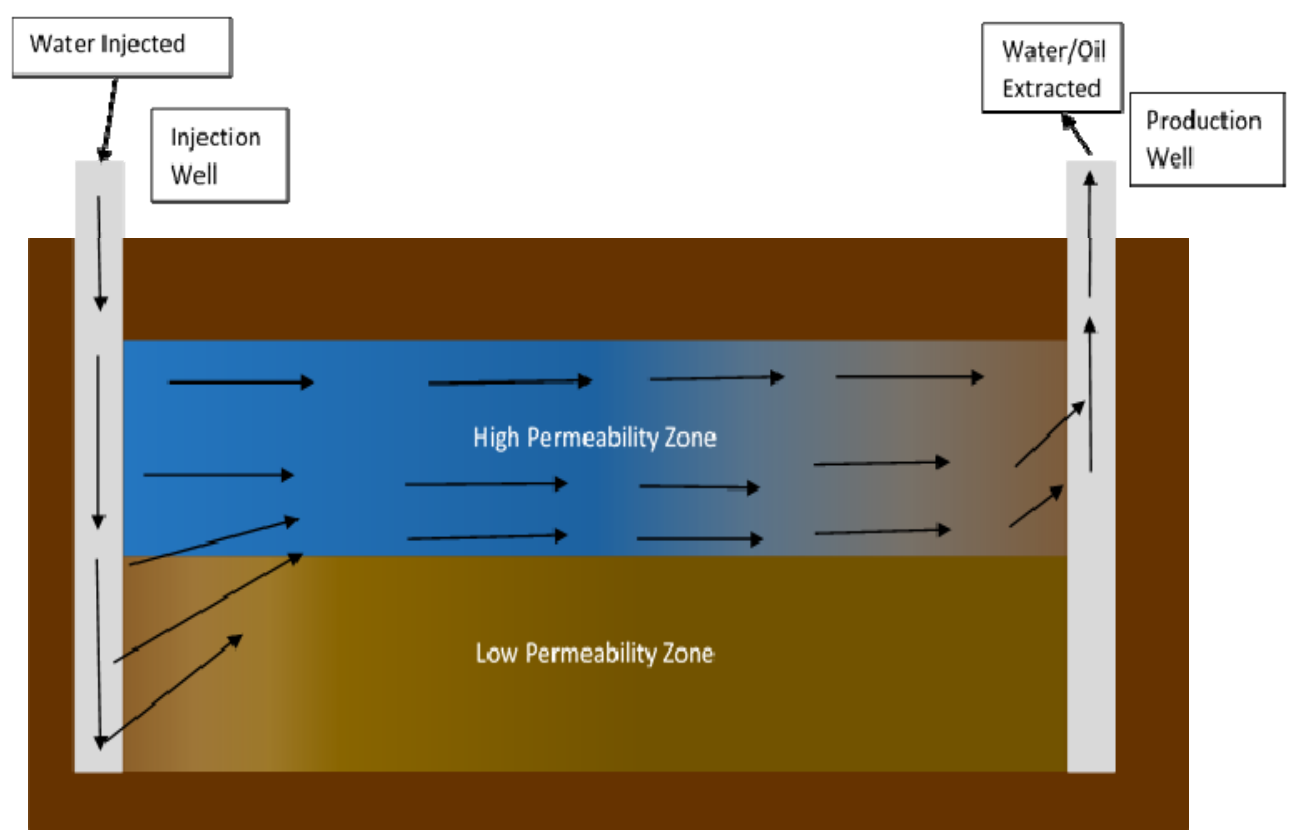

Fig. 1 Displays injected water movement though a heterogeneous, untreated reservoir.

permeability zone [10]. Doing so allows for a far more efficient and effective sweep of the low permeability zone, and therefore greater oil production, greater extraction efficiency, and longer well lives. However, unwanted water could also originate from the reservoir itself, as natural ground water sources. The isolation of such sources and zones could also enhance oil recovery and production.

Chemical methods, used extensively in decades, consist, specifically, of chemical products that are pumped into either production or injection wells [11, 12]. Chemical shut-off methods include: micro matrix cements, polymers micro particle blends, foamed systems, and particulate chemical blend gels/gelant.

Most polymer solutions, with time, evolve from low viscosity liquids to either strong or weak gels, depending on the solution's formulations. These gels can either partially or completely block the channels through which water is being produced. As a result, they are the basis of most WSO (water shut off) treatments. WSO treatments are frequently applied in fields with either waterflooding or polymer flooding. They allow sweeping pattern to be redirected to those areas that have not yet been produced.
An important number of WSO chemical methods have been developed in the last twenty years. Among these gel systems using both polyacrylamides and different crosslinkers have been the most extensively applied [13]. These crosslinkers can be classified as either inorganic or organic [14]. The inorganic crosslinking agents most widely used are based on $\mathrm{Cr}^{3+}$, $\mathrm{Al}^{3+}$, and $\mathrm{Zr}^{4+}$. These agents are used with PHPA (partially hydrolyzed polyacrilamides). These crosslinkers generate ionic bonds with the carboxilate (negatively charged) groups in polymer. Organic crosslinkers are more stable at high temperatures due to the covalent bonds generated with the PAM (polyacrylamide) amides groups [15].

One technique used to control excess water production in heterogeneous, permeable reservoirs is the use of an SAP (superabsorbent polymer). SAPs swell into a gel in the presence of water, and the ratio to which they swell is dependent upon the salinity, $\mathrm{pH}$, and solution temperature. This polymer is inserted into a reservoir and allowed to swell, decreasing the permeability of the high permeability zone while having little effect on the low permeability zone [16, 17]. This causes a decrease in reservoir heterogeneity, 
which will increase the ability of a water flooding operation to extract oil from the low permeability zone, and such an example can be seen in Fig. 2.

Such processes may also be used to completely isolate a zone. In order to best employ an SAP to a particular reservoir, it is important to understand the kinetics of the polymer so as to reduce formation damage, effectively target the high permeability zones, and efficiently remove the polymer, if desired.

The presence of divalent cations in a reservoir or brine can cause serious issues when some types of SAP are introduced to it, especially in solutions with $\mathrm{pH}$ greater than 3 to 4 [18]. As the polymer absorbs water and becomes a gel, divalent cations react catastrophically with the Sodium salt in the gelled polymer [18]. The issues caused by Calcium and other divalent cations can be mitigated by the use of a preflush in a reservoir, although such action may not be successful [1]. A similar effect can be observed in a reservoir that has been water flooded for an extended period of time [1]. The fact that these cations may remain begs the question: What is the effect of a mixed (monovalent ions and divalent ions) brine solution on an SAP, and how does that compare to brine solely composed of one or the other?

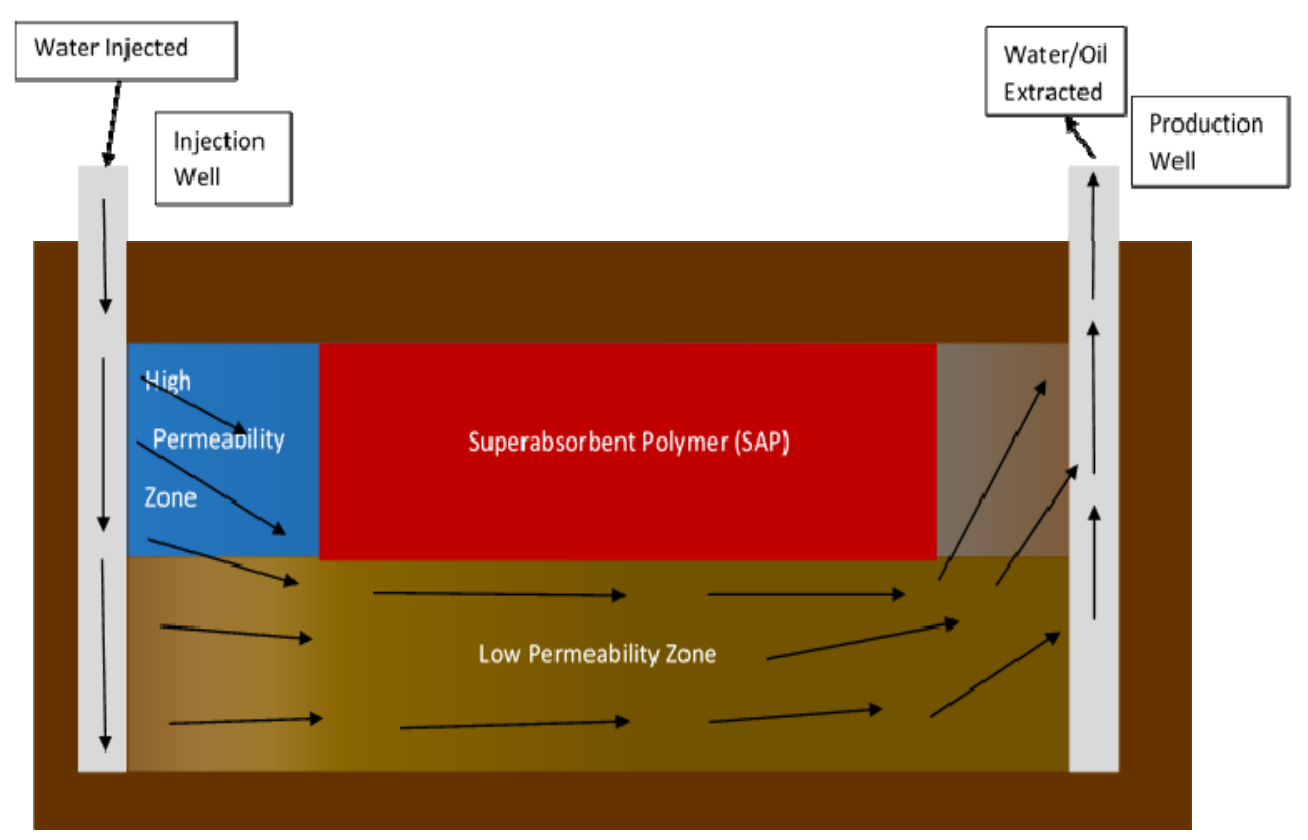

Fig. 2 Displays an example of a water flooding operation after successful SAP treatment.

\section{Experimental Work}

\subsection{Equipment and Materials}

\subsubsection{Equipment}

An electronic precision balance, a Tree ${ }^{\circledR}$ HRB103, was used to weigh out all components used in this work. In order to measure $\mathrm{pH}$ values, an Oakton ${ }^{\circledR} \mathrm{pH} 11$ series $\mathrm{pH}$ meter was used. In order to study the effect of temperature, a Julabo ${ }^{\circledR}$ F25 MC bath heater and circulator was employed. This device has the capability to both cool and heat samples inserted into the bath. To measure the volume of the polymer during experiments, the experiments were carried out in $15 \mathrm{~mL}$ graduated centrifuge tubes. In order to ensure that uniform and correct results were acquired, a VWR ${ }^{\circledR}$ Lab Dancer S41 agitator was used. The use of an agitator provided a repeatable way to expose the lowest SAP particles to the brine solution. An accurate reaction could then be recorded in a repeatable fashion.

\subsubsection{Brine}

To manufacture the brine, deionized (DI) water from was used. Sodium chloride, a popular salt in SAP kinetics studies, was mixed with the DI water by percent weight. Calcium chloride was also used both alone in a brine and in conjunction with sodium chloride. The 
Table 1 Salt concentration by brine type.

\begin{tabular}{ll}
\hline Brine type & Concentration ranges (by \% wt.) \\
\hline Sodium chloride & $1 \%, 2 \%, 5 \%, 10 \%, 12.5 \%, 15 \%, 17.5 \%, 20 \%$ \\
Calcium chloride & $1 \%, 1.5 \%, 2 \%, 3.5 \%, 5 \%, 10 \%$ \\
$\begin{array}{l}\text { Sodium chloride and } \\
\text { calcium chloride }\end{array}$ & $1 \% 1.5 \%, 2 \%, 3 \%, 5 \%, 10 \%, 12.5 \%,(90 \%$ of salt wt. was sodium chloride, $10 \%$ was calcium chloride.) \\
\hline
\end{tabular}

brines were mixed in a $15 \mathrm{~mL}$ graduated centrifuge tube.

\subsubsection{Polymer}

The polymer used was AT-O3S and Liquiblock 40F, a Sodium salt and a Potassium salt respectively of crosslinked polyacrylic acid. The particle sizes of both polymers ranged from 35 to 60 mesh or 250 to 500 microns. The SAP used in this project was sensitive to brine salinity, temperature, and $\mathrm{pH}$. These polymers have the ability to absorb large amounts of water.

\subsection{Procedure}

In order to study the kinetics of the polymer, it was allowed to swell and deswell over time in brines comprised of DI water, sodium chloride, calcium chloride, or a mix of sodium chloride and calcium chloride. The effect of temperature on the final swelling ratio was then investigated by adjusting the temperature of preswelled samples. All components were weighed out on an electronic precision balance prior to being combined in the graduated centrifuge tubes. The brine solutions were mixed by percent weight and agitated until all visible evidence of salt had vanished. The sodium chloride concentration ranged from $1 \%$ to $20 \%$, the calcium chloride concentration ranged from $1 \%$ to $10 \%$, and the concentration of the combination of the two (mixed brine) ranged from $1 \%$ to $12.5 \%$ in a ratio of 9 parts sodium chloride to 1 part calcium chloride, measured by weight, as seen in Table 1. This ratio was chosen based on reported reservoir salinities from Montana and North Dakota in the United States [19].

Due to precipitation issues, the concentration ranges of brines containing calcium chloride had to be limited in order to prevent wasteful experiments. The $\mathrm{pHs}$ and temperatures were measured prior to brine introduction to the polymer. After introducing the brine to the polymer, the tubes were immediately agitated on a test tube shaker. Agitation lasted until 30 seconds prior to taking a reading, unless the time between readings was greater than or equal to 5 minutes. In the latter case, 1 minute was given for the polymer to settle so an accurate reading could be taken. The readings were taken in $\mathrm{mL}$ by evaluating the volume of the polymer using the graduated centrifuge tubes.

\subsubsection{Polymer Swelling}

The AT-O3S was weighed out ( $1 \%$ by brine weight) and added to a separate, dry tube. The brine was mixed, then it was added to polymer in a dry tube, and agitated. Swelling was initially measured by the volume of the polymer. The following Eq. (1), where, $S$ is the swelling ratio, $V$ is the volume of the polymer at the time of measurement, and $V_{\mathrm{o}}$ is the volume of the polymer in powder form,

$$
S=\frac{V-V_{0}}{V_{0}}
$$

provided the swelling ratio. In order to find the initial volume of the polymer, the density of the polymer had to be considered. Using the mass and density, the following equation was used to find $V_{\mathrm{o}}$.

$$
V_{0}=\frac{m_{p}}{\rho_{p}}
$$

where, $m_{p}$ is the initial mass of the polymer and $\rho_{p}$ is the density of the polymer. The original density used for the polymer was $1.23 \mathrm{~g} / \mathrm{mL}$.

\subsubsection{Polymer Deswelling}

The SAP was weighed out at $0.1 \%$ by percent weight of the DI water and added to a dry centrifuge tube. $15 \mathrm{~g}$ of DI water was then added to the polymer and it was agitated until fully swollen. Care was taken to allow a 
layer of water to remain free at the top of the tube to allow for quick dissolution of the salts, giving a more accurate curve. Deswelling was measured by the volume of the polymer then converted into swelling ratio using Eqs. (1) and (2).

\subsubsection{Polymer Temperature Sensitivity}

Previously swelled and deswelled SAP was placed in a heated and refrigerated circulator (Julabo® F25 MC) in order to control the temperature which was varied from $5{ }^{\circ} \mathrm{C}$ to $90{ }^{\circ} \mathrm{C}$. The tubes were allowed to reach the desired temperature, which was confirmed by the pH 11 meter. The volume of the polymer was then recorded and converted to swelling ratio using the same methods as above.

\section{Results and Discussion}

\subsection{Salinity Effect}

\subsubsection{Sodium Chloride Effect}

The swelling and deswelling curves of the chosen polymer in Sodium Chloride brines showed very smooth curves as shown in Figs. 3a and 3b. Swelling and deswelling occurred quickly, reaching the final swelling ratio within 10 minutes in most instances. Swelling and deswelling ratios for equal brine concentrations approached similar values and the final ratio decreased as salinity increased, which is displayed in Fig. 4. The $\mathrm{pH}$ for Sodium Chloride brines tended to be slightly acidic, as seen in Table 2 .

Table 2 pH of salt brines by concentration.

\begin{tabular}{llllllllllll}
\hline Concentration (\% wt.) & $1 \%$ & $1.5 \%$ & $2 \%$ & $3 \%$ & $3.5 \%$ & $5 \%$ & $10 \%$ & $12.5 \%$ & $15 \%$ & $17.5 \%$ & $20 \%$ \\
\hline pH sodium chloride & 6.2 & - & 5.8 & - & - & 5.8 & 5.9 & 6.6 & 6.4 & 6.6 & 6.6 \\
pH calcium chloride & 9.2 & 9.5 & 9.9 & - & 9.9 & 9.5 & 9.7 & - & - & - & - \\
pH mixed brines & 8.3 & 8.5 & 9 & 8.8 & - & 9.2 & 9.4 & 9.2 & - & - & - \\
\hline
\end{tabular}

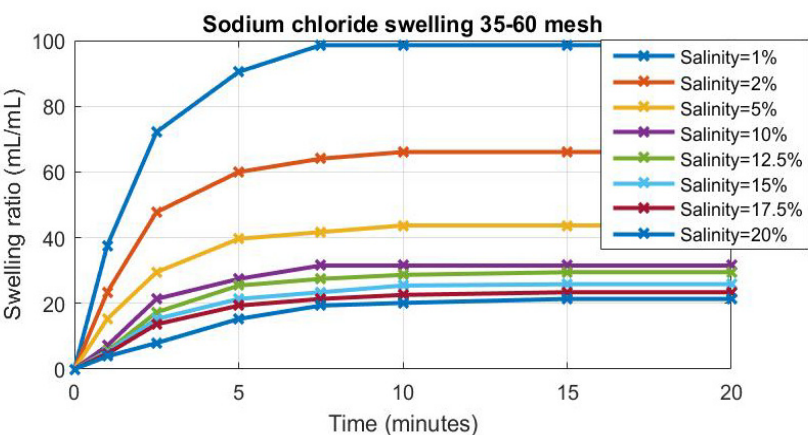

(a)

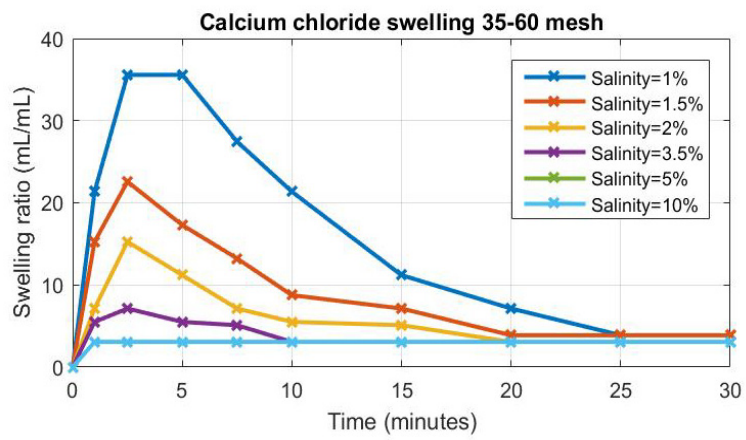

(c)

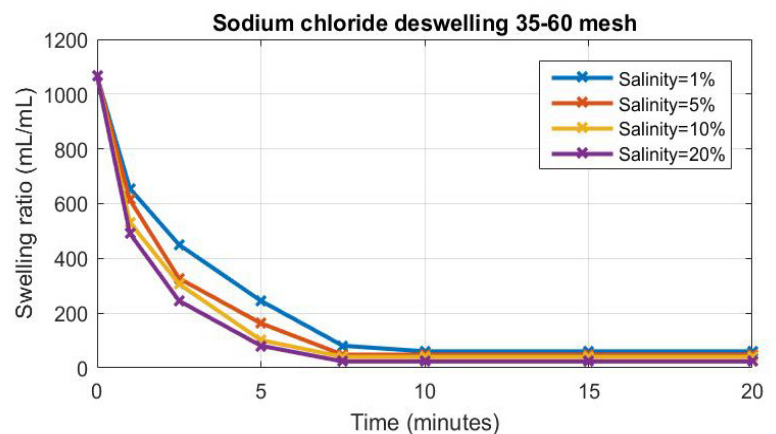

(b)

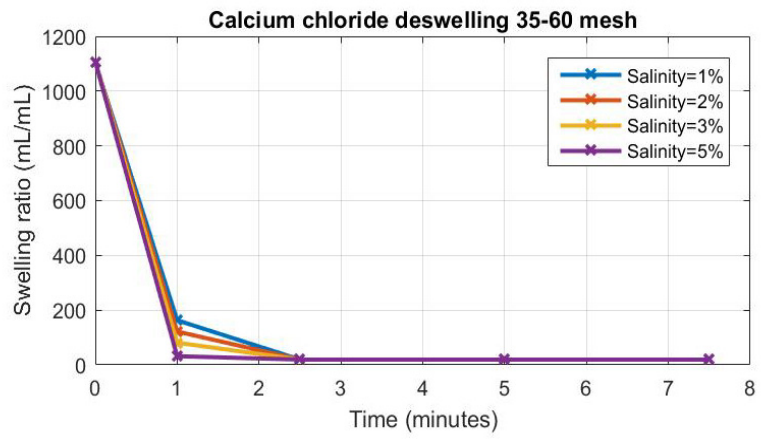

(d)

Fig. 3 (a) $1 \%$ polymer swelling in various sodium chloride brines; (b) $0.1 \%$ polymer deswelling in various sodium chloride brines; (c) $1 \%$ polymer swelling in various calcium chloride brines; (d) $0.1 \%$ polymer deswelling in various calcium chloride brines. 


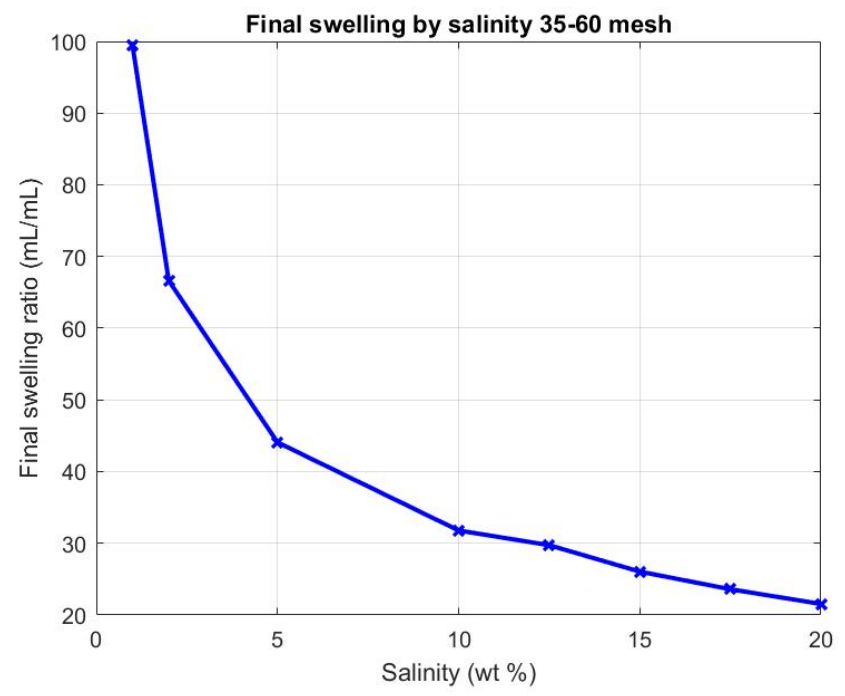

Fig. 4 Final swelling volume of polymer in sodium chloride brine by salinity.

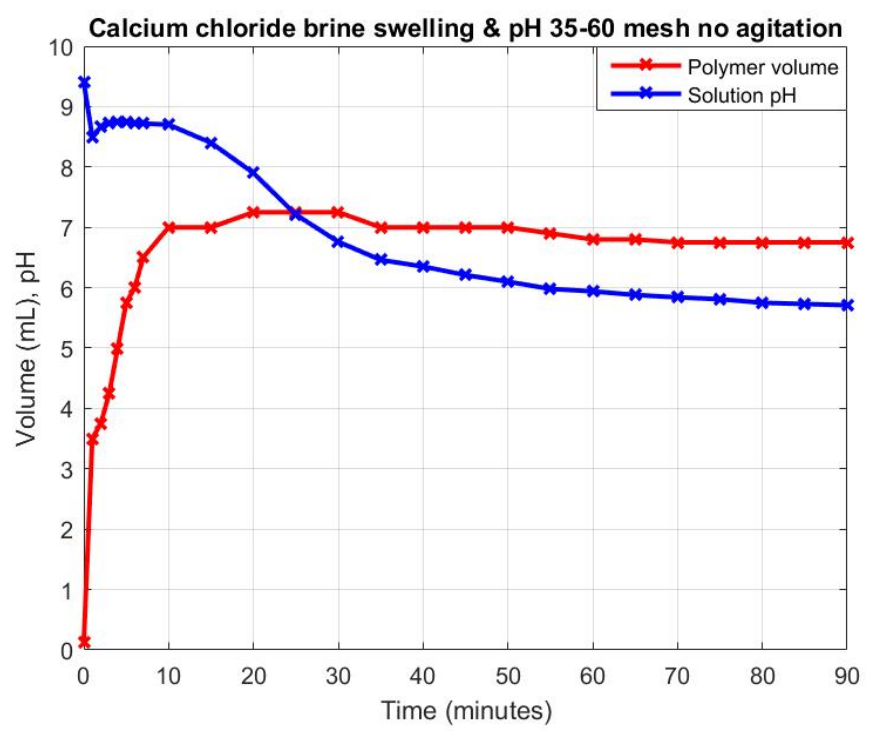

Fig. 5 Polymer volume and $\mathrm{pH}$ of AT-O3S in $1 \%$ calcium chloride brine by time.

\subsubsection{Calcium Chloride Effect}

The chosen SAP reacted poorly when it was exposed to a Calcium Chloride brine, as seen in Figs. $3 \mathrm{c}$ and $3 \mathrm{~d}$. As the polymer gelled, Calcium reacted with the Sodium salt present in the polymer forming Calcium polyacrylate. The insoluble product would then fall out of solution as a white precipitate. This gave the appearance of deswelling in readings taken during swelling experiments. However, during a swelling attempt displayed in Fig. 5, the polymer was not agitated for the first 90 minutes. It is inferred that this polymer failed to precipitate out dramatically due to the combination of two factors. One factor was inhibition of contact between the gel and the solution due to the formation of a precipitate layer on the surface of the gel and a decrease in the $\mathrm{pH}$ of the solution as the gel increased in volume. The gels are slightly acidic, tending to range from 6 to $7 \mathrm{pH}$, so as the polymer gels, it tends to drop the $\mathrm{pH}$ of the calcium chloride brines. This takes time to occur and cannot happen quickly enough when the gel is agitated. With agitation, the surface area of the gel exposed to the brine increases and the areas of higher $\mathrm{pH}$ are allowed to come in contact with gel readily. This is likely the 
cause of the accelerated precipitation seen in the agitated gels. The $\mathrm{pH}$ of the calcium chloride brines prior to SAP introduction varied between 9 and 10 as seen in Table 2. Attempts to deswell resulted in complete precipitation of the polymer within 2 minutes as seen in Fig. 3d.

3.1.3 Mixed Brine Effect

When the SAP was introduced to a mixed brine of

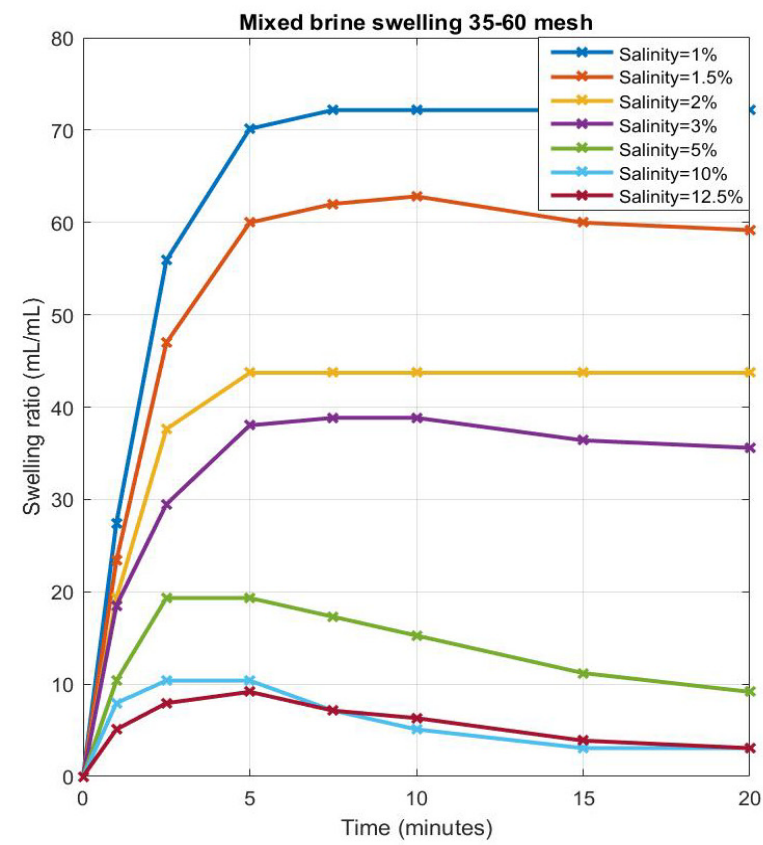

(a) sodium chloride and calcium chloride, the polymer would fail to precipitate out fully during swelling, until reaching a total brine concentration of approximately $10 \%$ as seen in Figs. $6 \mathrm{a}$ and $6 \mathrm{~b}$. The $\mathrm{pH}$ of the brines tended to vary between 8.5 and 9.5 as shown in Table 2 . During deswelling, all brine concentrations fully precipitated out above $1 \%$. This occurred within 2 minutes of the polymer's first contact with the brine.

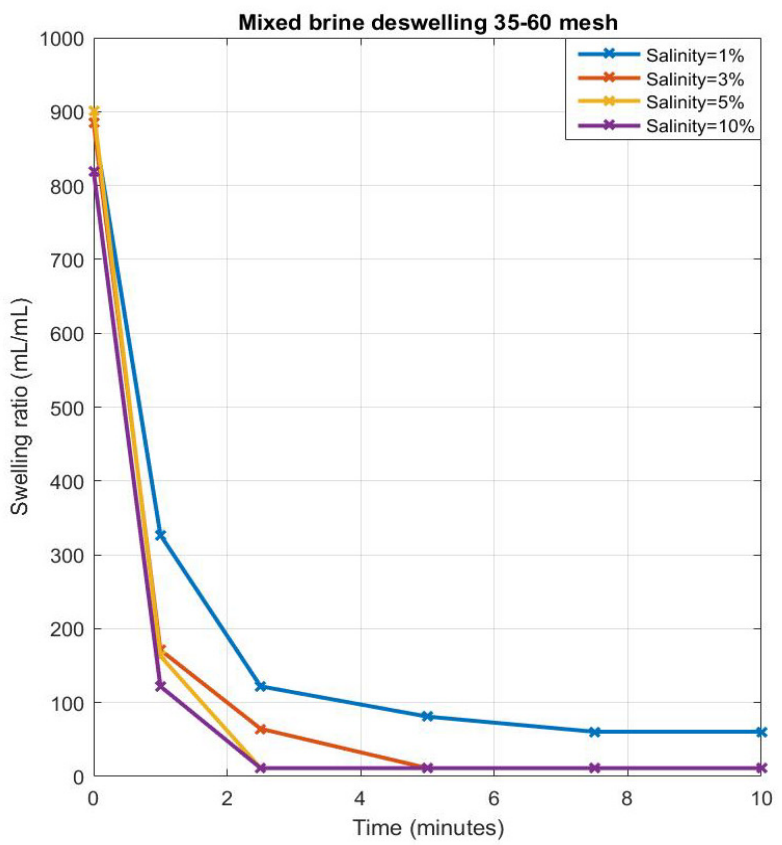

(b)

Fig. 6 (a) $1 \%$ polymer swelling in various mixed brines $\left(9\right.$ to 1 ratio of $\mathrm{NaCl}$ to $\left.\mathrm{CaCl}_{2}\right)$; (b) $0.1 \%$ polymer deswelling in various mixed brines ( 9 to 1 ratio of $\mathrm{NaCl}$ to $\mathrm{CaCl}_{2}$ ).

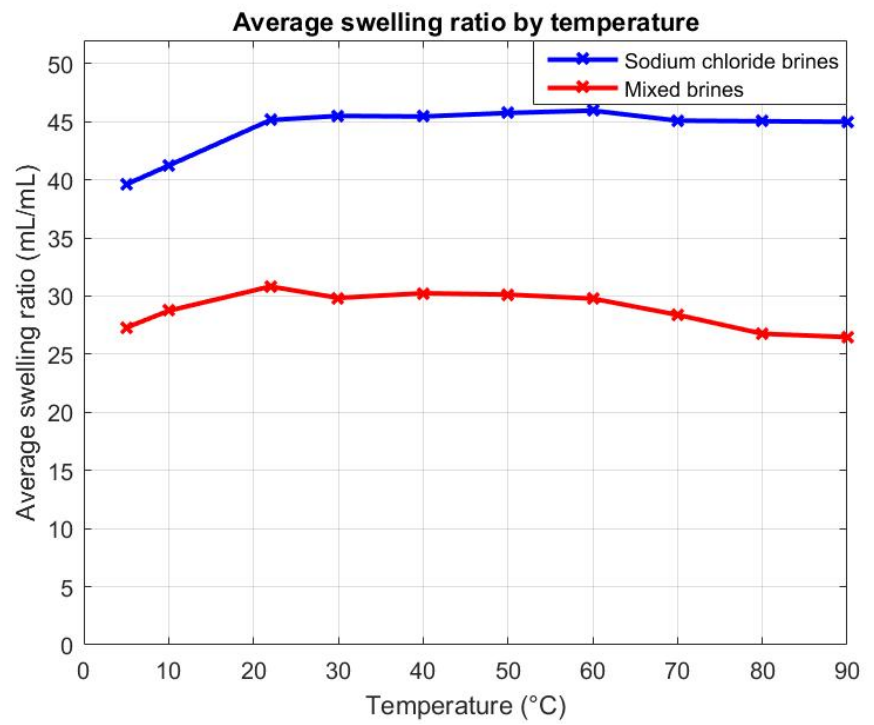

Fig. 7 Average swelling ratio for brine type by temperature in degrees celsius for AT-O3S in sodium chloride and mixed brines. Room temperature was $22^{\circ} \mathrm{C}$. 


\subsection{Temperature Effects}

The temperature results, in Fig. 7, showed a clear decrease in the swelling ratios of the SAP in both the Sodium Chloride brines and the mixed brines as the temperature decreased.

Results from calcium chloride brines were not included in the results, as samples fully precipitated out at every concentration, resulting in nothing but insoluble precipitate. For the same reason, the $10 \%$ and $12.5 \%$ mixed brines were also omitted. As the temperature increased, the results were unclear for the sodium chloride brines, which increased very slowly on average until about $60{ }^{\circ} \mathrm{C}$, at which point the average volume decreased slightly. For the SAP swollen in mixed brines, the swelling ratio decreased as temperature increased due to further precipitation of the polymer and calcium.

\section{Conclusions}

(1) The SAP's reaction to sodium chloride brines appeared to be very predictable based on prior knowledge, as its final swelling ratios declined as salinity increased and the ratios for both swelling and deswelling approached similar values.

(2) Calcium chloride reacted with the gelled polymer's sodium salt, forming calcium polyacrylate, resulting in complete destruction of the polymer chain over the majority of the salinity range.

(3) The mixed brines showed much greater stability when compared to calcium chloride brines, and when compared to sodium chloride brines, mixed brines had a much lower swelling ratio and a much greater, irreversible sensitivity to temperature changes.

(4) During deswelling, precipitation appeared to be accelerated. This may be due to the inability of the polymer to decrease the $\mathrm{pH}$ of the solution before the gel made significant contact with present divalent cations in solution.

(5) The data suggest that the use of acid washes and/or preflushes before the implementation of an SAP treatment in reservoirs containing significant amounts of divalent cations is of great importance to the success of an operation, even if their concentration is relatively small.

(6) Adjustment for the presence of divalent cations should be considered in the event that preflush or acid wash operations fail in order to increase SAP treatment success.

(7) The protection of already swollen gel in a reservoir containing divalent cations becomes a matter of great importance, as destruction of the gel can happen relatively quickly even in low salinity circumstances. This issue is amplified in high temperature reservoirs.

\section{Acknowledgments}

This project could not have been possible without the following contributors. The Midwestern State University EURECA program graciously provided the monetary support for the project. Lab space was provided by the McCoy School of Engineering at Midwestern State University, and some supplies and equipment was loaned by the Midwestern State University Chemistry Department. Dr. Jianguo Shao of the Midwestern State University Chemistry Department provided insight on the reaction between Calcium and the polymer's crosslinker which was incredibly helpful. Thank you to all contributors for the support and assistance from start to finish, the project could not have been completed without the support.

\section{References}

[1] Sheng, J. J., Leonhardt, B., and Azri, N. 2015. "Status of Polymer-Flooding Technology." Journal of Canadian Petroleum Technology 54 (2): 116-26.

[2] Xu, W., Ok, J. T., Xiao, F., Neeves, K. B., and Yin, X. 2014. "Effect of Pore Geometry and Interfacial Tension on Water-Oil Displacement Efficiency in Oil-Wet Microfluidic Porous Media Analogs." Physics of Fluids 26 (9): paper 093102.

[3] Dalrymple, E. D. 1997. "P14 Water Control Treatment Design Technology." Presented at the 15th World Petroleum Congress, Beijing, China. 
[4] Veil, J. A., Puder, M. G., Elcock, D., Robert, J., and Redweik, J. A. 2004. White Paper Describing Produced Water from Production of Crude Oil, Natural Gas, and Coal Bed Methane. Technical report prepared for National Energy Technology Library (U.S. DOE, under Contract No. W-31-109-Eng-38), Argonne National Laboratory, Argonne, Illinois.

[5] McCain, W. D. Jr. 1990. The Properties of Petroleum Fluids. 2nd edition, Tulsa, OK: PennWell, 438-9.

[6] Bailey, B., Crabtree, M., Elphick, J., Kuchuk, F., Romano, C., and Roodhart, L. 2000. "Water Control." Oilfield Review, 30.

[7] Elsharafi, M. O. 2015. "Effect of Back Pressure on Gel Pack Permeability during Conformance Control Treatment." Presented at the 2015 Southwest Section of the AAPG Annual Convention held in Wichita Falls, Texas.

[8] Elsharafi, M., and Bai, B. 2015. "Gel Pack-A Novel Concept to Optimize Preformed Particle Gel Conformance Control Treatment Design." In Proceedings of the 62nd Southwestern Petroleum Conference, 275-86. doi:10.2118/38039-MS.

[9] Chia, P. C., and Bai, B. 2010. "Modeling Preformed Particle Gel Swelling and Deswelling Kinetics." Undergraduate Research at Missouri School of Technology, Department of Geological Sciences and Engineering.

[10] Needham, R. B., and Doe, P. H. 1987. "Polymer Flooding Review." Society of Petroleum Engineers (SPE), SPE paper 17140.

[11] Sydansk, R. D., and Southwell, G. P. 2000. "More than 12 Years' Experience with a Successful Conformance Control Polymer Gel Technology." SPE Production \& Facilities 15 (4): 270-8. doi: 10.2118/66558-PA.

[12] Seright, R. S., Lane, R. H., and Sydansk, R. D. 2003. “A
Strategy for Attacking Excess Water Production." SPE Production \& Facilities 18 (3): 158-69. doi: 10.2118/84966-PA.

[13] Vega, I., Morris, W., Robles, J., Peacock, H., and Marin, A. 2010. "Water Shut-off Polymer Systems: Design and Efficiency Evaluation Based on Experimental Studies." In Proceedings of the Improved Oil Recovery Symposium, 1-18. doi: 10.2118/129940-MS.

[14] Al-Muntasheri, G. A., Nasr-El-Din, H. A., Peters, J. A., and Zitha, P. L. J. 2006. "Investigation of a High Temperature Organic Water Shut-off Gel: Reaction Mechanisms." SPE Journal 11 (4): 497-504. doi: 10.2118/97530-PA.

[15] Al-Muntasheri, G. A., Nasr-El-Dir, M. A., Al Noaimi, K. R., and Zitha, P. L. J. 2007. "A Study of Polyacrylamide-Based Gels Crosslinked with Polyethyleneimine." In Proceedings of the SPE International Symposium on Oilfield Chemistry, 1-9. doi: 10.2118/105925-PA.

[16] Elsharafi, M. O., and Bai, B. 2012. "Effect of Weak Preformed Particle Gel on Unswept Oil Zones/Areas during Conformance Control Treatments." Industrial \& Engineering Chemistry Research 51 (35): 11547-53.

[17] Elsharafi, M. O., and Bai, B. 2016. "Influence of Strong Preformed Particle Gels on Low Permeable Formations in Mature Reservoirs." Petroleum Science 13 (1): 77-90.

[18] Al-Anazi, H. A., Aramco, S., and Sharma, M. M. 2002. "Use of a pH Sensitive Polymer for Conformance Control." Presented at the SPE International Symposium and Exhibition on Formation Damage Control held in Lafayette, Louisiana.

[19] Dandekar, A. Y. 2013. Petroleum Reservoir Rock and Fluid Properties, 2nd Edition. CRC Press. 6000 Broken Sound Parkway NW, Suite 300 Boca Raton, FL. 